\title{
Asymptotic behavior of a fault diagnosis performance measure for linear systems
}

Jan Åslund, Erik Frisk and Daniel Jung

The self-archived postprint version of this journal article is available at Linköping University Institutional Repository (DiVA):

http://urn.kb.se/resolve?urn=urn:nbn:se:liu:diva-158919

N.B.: When citing this work, cite the original publication.

Åslund, J., Frisk, E., Jung, D., (2019), Asymptotic behavior of a fault diagnosis performance measure for linear systems, Automatica, 106, 143-149. https://doi.org/10.1016/j.automatica.2019.04.041

Original publication available at:

https://doi.org/10.1016/j.automatica.2019.04.041

Copyright: Elsevier

http://www.elsevier.com/

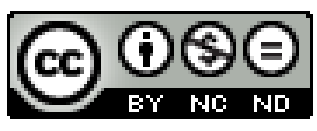




\title{
Asymptotic behavior of a fault diagnosis performance measure for linear systems
}

\author{
Jan Åslund, Erik Frisk, and Daniel Jung \\ Linköping University, Linköping, Sweden
}

\begin{abstract}
Fault detection and fault isolation performance of a model based diagnosis system mainly depends on the level of model uncertainty and the time allowed for detection. The longer time for detection that can be accepted, the more certain detection can be achieved and the main objective of this paper is to show how the window length relates to a diagnosis performance measure. A key result is an explicit expression for asymptotic performance with respect to window length and it is shown that there exists a linear asymptote as the window length tends to infinity. The gradient of the asymptote is a system property that can be used in the evaluation of diagnosis performance when designing a system. A key property of the approach is that the model of the system is analyzed directly, which makes the approach independent of detection filter design.
\end{abstract}

Key words: Fault diagnosability analysis, Fault detection and isolation, Model-based diagnosis, Asymptotic analysis.

\section{Introduction}

A quantitative fault diagnosability performance measure for fault detection and isolation of time-discrete linear descriptor models with Gaussian distributed noise has been proposed in [2] and [3]. The measure, called distinguishability in [2], quantifies diagnosability performance given a model and takes process noise, measurement noise, and fault time profiles into consideration and gives an upper limit of achievable fault-to-noise ratio of any linear residual generator generated from that model.

The asymptotic behavior of the distinguishability measure, when the number of samples goes to infinity, gives useful information about the fault detection and isolation properties of the system. In this paper, an expression of the distinguishability asymptote, as function of window length, is derived. It is shown that there exists a linear asymptote as the window length tends to infinity and it is also shown how to compute the asymptote.

\footnotetext{
* This paper was not presented at any IFAC meeting. Corresponding author J. Åslund. Tel. +46 13 281692. Fax +46 13282035 .

Email addresses: jan.aslund@liu.se (Jan Åslund), erik.frisk@liu.se (Erik Frisk), daniel.jung@liu.se (Daniel Jung).
}

\section{Problem formulation}

Before stating the problem formulation, a brief summary of the definition of the distinguishability measure in [2] is presented. Consider a time-discrete linear model in the form

$$
\begin{aligned}
\mathbf{x}_{t+1} & =A \mathbf{x}_{t}+B^{u} \mathbf{u}_{t}+B^{f} \mathbf{f}_{t}+\mathbf{v}_{t} \\
\mathbf{y}_{t} & =C \mathbf{x}_{t}+D^{u} \mathbf{u}_{t}+D^{f} \mathbf{f}_{t}+\varepsilon_{t}
\end{aligned}
$$

where $\mathbf{x} \in \mathbb{R}^{n}$ are state variables, $\mathbf{y} \in \mathbb{R}^{n_{y}}$ are measured signals, $\mathbf{u} \in \mathbb{R}^{n_{u}}$ are input signals, $\mathbf{f} \in \mathbb{R}^{n_{f}}$ are modeled faults, $\mathbf{v}_{t}$ and $\varepsilon_{t}$ are i.i.d. Gaussian random vectors with zero mean. Without loss of generality, identity covariance matrices are assumed. Furthermore, it is assumed that the matrix $A$ is invertible.

Define the model vectors on a window of size $N$ as

$$
\begin{aligned}
& \mathbf{z}=\left(\mathbf{y}_{t-n+1}^{T}, \ldots, \mathbf{y}_{t}^{T}, \mathbf{u}_{t-n+1}^{T}, \ldots, \mathbf{u}_{t}^{T}\right)^{T} \\
& \mathbf{x}=\left(\mathbf{x}_{t-n+1}^{T}, \ldots, \mathbf{x}_{t}^{T}, \mathbf{x}_{t+1}^{T}\right)^{T} \\
& \mathbf{f}=\left(\mathbf{f}_{t-n+1}^{T}, \ldots, \mathbf{f}_{t}^{T}\right)^{T} \\
& \mathbf{e}=\left(\mathbf{v}_{t-n+1}^{T}, \ldots, \mathbf{v}_{t}^{T}, \varepsilon_{t-n+1}^{T}, \ldots, \varepsilon_{t}^{T}\right)^{T}
\end{aligned}
$$

Then, a sliding window model of length $N$ can be written 
as $L \mathbf{z}=H \mathbf{x}+F \mathbf{f}+\mathbf{e}$ where

$$
H=\left(\begin{array}{ccccc}
A & -I & 0 & \ldots & 0 \\
0 & A & -I & & 0 \\
\vdots & & \ddots & \ddots & \vdots \\
0 & 0 & \ldots & A & -I \\
C & 0 & 0 & \ldots & 0 \\
0 & C & 0 & & 0 \\
\vdots & & \ddots & & \vdots \\
0 & 0 & \ldots & C & 0
\end{array}\right), F=\left(\begin{array}{cccc}
B_{f} & 0 & \ldots & 0 \\
0 & B_{f} & & 0 \\
\vdots & & \ddots & \vdots \\
0 & 0 & \ldots & B_{f} \\
D_{f} & 0 & \ldots & 0 \\
0 & D_{f} & & 0 \\
\vdots & & \ddots & \vdots \\
0 & 0 & \ldots & D_{f}
\end{array}\right)
$$

and $F^{i}$ and $F^{j}$ denote the columns in $F$ corresponding to fault $f^{i}$ and $f^{j}$, respectively.

Then, define the random variable $\xi=\mathcal{N}_{H} L \mathbf{z}$ where the set of rows of $\mathcal{N}_{H}$ is an orthonormal basis for the left nullspace of $H$. Note that the distribution of $\xi$ depends on $\mathbf{f}$ but is independent of $\mathbf{x}$. Let $p\left(\xi ; \mathcal{N}_{H} F f\right)$, denote a multivariate probability density function, pdf, with mean $\mathcal{N}_{H} F f$ and identity covariance matrix describing $\xi$. To model all possible realizations of a fault $f^{i}$, let $\mathcal{Z}_{f^{i}}$ denote the set of all pdfs $p(\xi ; \mu(\theta))$, for all fault time profiles $\theta \in \mathbb{R}^{N}$, describing $\xi$ which could be explained by the fault $f^{i}$, i.e., $\mathcal{Z}_{f^{i}}=\left\{p\left(\xi ; \mathcal{N}_{H} F^{i} \theta\right) \mid \forall \theta \in \mathbb{R}^{N}\right\}$. A specific fault time profile $f^{i}=\theta$ is denoted $p_{\theta}^{i}=p\left(\xi ; \mathcal{N}_{H} F^{i} \theta\right)$. Then, the distinguishability measure is defined as follows.

Definition 1 (Distinguishability) Given a sliding window model, distinguishability $\mathcal{D}^{i, j}(\theta)$ of a fault $f^{i}$ with a given fault time profile $\theta$ from a fault mode $f^{j}$ is defined as $\mathcal{D}^{i, j}(\theta)=\min _{p^{j} \in \mathcal{Z}_{f j}} K\left(p_{\theta}^{i} \| p^{j}\right)$ where $K\left(p^{i} \| p^{j}\right)$ is the Kullback-Leibler divergence from $p d f p^{i}$ to $p^{j}$.

Distinguishability measures the minimum KullbackLeibler divergence between observation pdf with fault time profile $f^{i}=\theta$ from fault mode $f^{j}$. Thus, distinguishability depends on the window length $N$ and fault time profile $\theta \in \mathbb{R}^{N}$ and quantifies how easy it is to detect and isolate the modeled faults. A higher distinguishability value corresponds to a fault that is easier to detect or isolate.

To introduce the problem formulation, a small example is considered which will also be used throughout this paper. Consider the following time-discrete linear state space model

$$
\begin{aligned}
\mathbf{x}_{t+1} & =\left(\begin{array}{ccc}
1 & 0 & 0.3 \\
-0.2 & -1 & 0.4 \\
-0.1 & 0 & 0.7
\end{array}\right) \mathbf{x}_{t}+\left(\begin{array}{c}
0.3 \\
0.6 \\
-0.6
\end{array}\right) \mathbf{u}_{t}+\left(\begin{array}{lll}
0 & 2 & 0 \\
0 & 0 & 1 \\
0 & 0 & 0
\end{array}\right) \mathbf{f}_{t}+\mathbf{v}_{t} \\
\mathbf{y}_{t} & =\left(\begin{array}{ccc}
0.2 & 0 & 0 \\
0 & 0.8 & 0 \\
0 & 0 & 0.2
\end{array}\right) \mathbf{x}_{t}+\left(\begin{array}{l}
0 \\
0 \\
0
\end{array}\right) \mathbf{u}_{t}+\left(\begin{array}{lll}
1 & 0 & 0 \\
0 & 1 & 0 \\
0 & 0 & 0
\end{array}\right) \mathbf{f}_{t}+\varepsilon_{t}
\end{aligned}
$$

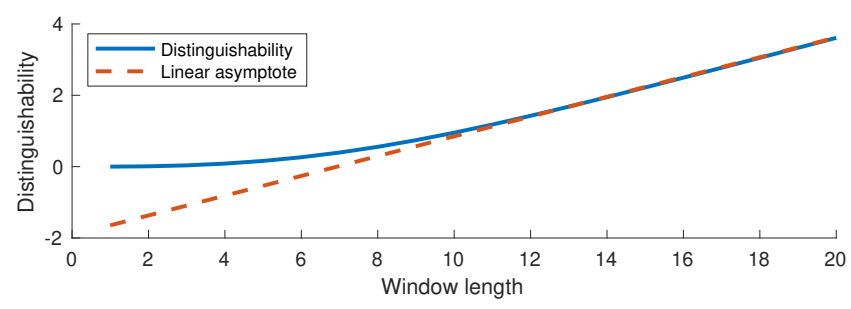

Fig. 1. Distinguishability (solid line), as function of window length, for isolating fault $f^{2}$ from fault $f^{3}$, and the linear asymptote (dashed line)

where $\mathbf{x}$ are states, $\mathbf{y}$ sensors, $\mathbf{u}$ known inputs, $\mathbf{f}$ faults, and $\mathbf{v}_{t} \sim \mathcal{N}\left(0, \Sigma_{\mathbf{v}}\right)$ and $\varepsilon_{t} \sim \mathcal{N}\left(0, \Sigma_{\varepsilon}\right)$ are i.i.d. Gaussian noise where the covariance matrices $\Sigma_{\mathbf{v}}$ and $\Sigma_{\varepsilon}$ are identity matrices.

Distinguishability is first evaluated for the time window length, $N=12$, and the faults to be detected and isolated are assumed to have constant magnitude equal to one during the time window, i.e., $\theta=(1,1, \ldots, 1)^{T}$. Distinguishability is computed for each fault pair and the results are shown in Table 1 . A positive value at position $(i, j)$ corresponds to that fault $f^{i}$ is isolable from fault $f^{j}$, or detectable if isolable from the No Fault (NF) case.

Table 1

Distinguishability for the model (3) given a time window $N=12$ and the gradients of the asymptotes are given in parenthesis.

\begin{tabular}{ccccc}
\hline & $N F$ & $f^{1}$ & $f^{2}$ & $f^{3}$ \\
\hline$f^{1}$ & $1.17(0.13)$ & $0.00(0.00)$ & $0.35(0.05)$ & $0.37(0.06)$ \\
$f^{2}$ & $3.90(0.44)$ & $1.11(0.15)$ & $0.00(0.00)$ & $1.42(0.28)$ \\
$f^{3}$ & $0.56(0.05)$ & $0.15(0.03)$ & $0.18(0.03)$ & $0.00(0.00)$ \\
\hline
\end{tabular}

The table shows that it is, e.g., easier to detect $f^{2}$ than $f^{3}$, since $3.90>0.56$. Note that distinguishability is not a symmetric relation, e.g., it is easier to isolate $f^{2}$ from $f^{3}$ than vice versa, since $1.42>0.18$. This non-symmetry will be investigated in this paper and it will be shown that this is a consequence of the fact that it is easier to detect $f^{2}$ than $f^{3}$ and an asymptotic result will be given in Corollary 1 in Section 3 as well as a geometric interpretation.

Distinguishability increases as the window length increases and in Fig. 1 distinguishability for isolating $f^{2}$ from $f^{3}$ is shown as a function of window length. In this paper the asymptotic behavior of distinguishability as a function of window length will be studied and the main result is that there exists a linear asymptote as $N$ tends to infinity; see Theorem 1 and Theorem 2 in Section 3. The gradients for model (3) are shown in parenthesis in Table 1.

Distinguishability $\mathcal{D}^{i, j}(\theta ; N)$ for isolating fault $f^{i}$, with a given fault time profile $\theta^{i}$ from fault $f^{j}$, with arbitrary 
fault time profile $\theta^{j}$, is computed by solving the least squares problem [2]

$$
\mathcal{D}^{i, j}(\theta ; N)=\min _{\mathbf{x}_{b}, \theta^{j}} \frac{1}{2}\left\|H \mathbf{x}_{b}+F^{j} \theta^{j}-F^{i} \theta\right\|^{2}
$$

Distinguishability can be computed explicitly by first eliminating the unknown variables. As an example, fault detection performance of fault $f_{i}$, denoted $\mathcal{D}^{i}(\theta ; N)$, can be computed as

$$
\mathcal{D}^{i}(\theta ; N)=\frac{1}{2}\left\|\mathcal{N}_{H} F^{i} \theta^{i}\right\|^{2}=\min _{\mathbf{x}_{b}} \frac{1}{2}\left\|H \mathbf{x}_{b}-F^{i} \theta^{i}\right\|^{2} .
$$

where the set of rows of $\mathcal{N}_{H}$ is an orthonormal base for the left null space of $H$. For further details and interpretations of distinguishability and related issues, see $[1,2]$.

\section{Asymptotic performance of distinguishability}

In this section the main results of the paper are presented. The proofs are given in Section 6. It is assumed in the asymptotic analysis that the fault is constant and it is sufficient to consider the constant fault time profile $\theta=(1,1, \ldots, 1)^{T}$, since distinguishability for an arbitrary fault size $\lambda$ is given by $\mathcal{D}^{i}(\lambda \theta, N)=\lambda^{2} \mathcal{D}^{i}(\theta, N)$ or $\mathcal{D}^{i, j}(\lambda \theta, N)=\lambda^{2} \mathcal{D}^{i, j}(\theta, N)$. The main result for asymptotic behavior of the detectability performance measure is the following.

Theorem 1 There exist constants $k^{i}, m^{i}$, and $0<\varrho<1$ such that

$$
\mathcal{D}^{i}(\theta, N)=k^{i} N+m^{i}+\mathcal{O}\left(\varrho^{N}\right)
$$

where $\theta=(1,1, \ldots, 1)^{T}$. If the system is observable, then the gradient $k$ of the linear asymptote is given by

$$
\begin{aligned}
k^{i} & =\min _{\mathbf{x} \in \mathbb{R}^{n}} \frac{1}{2}\left(\left\|\mathbf{x}-A \mathbf{x}-B_{f}^{i}\right\|^{2}+\left\|C \mathbf{x}+D_{f}^{i}\right\|^{2}\right) \\
& =\frac{1}{2}\left\|\mathcal{N}_{P}\left(\begin{array}{c}
B_{f}^{i} \\
D_{f}^{i}
\end{array}\right)\right\|^{2},
\end{aligned}
$$

where the set of rows of $\mathcal{N}_{P}$ is an orthonormal basis for the left null-space of $P=\left((A-I)^{T} C^{T}\right)^{T}$.

If the system is not observable, the non-observable part of the system has to be factored out before the theorem is applied, see Chapter 2.4 in [4].

Now the isolability performance is considered. In the next section it will be shown that for given $j$ there exists a reduced model, not depending on $f^{j}$, such that $\mathcal{D}^{i, j}$ for the original model is equal to $\mathcal{D}^{i}$ for the reduced model. A consequence is the following result.
Theorem 2 There exist constants $k^{i, j}, m^{i, j}$, and $0<$ $\varrho<1$ such that

$$
\mathcal{D}^{i, j}(\theta ; N)=k^{i, j} N+m^{i, j}+\mathcal{O}\left(\varrho^{N}\right)
$$

where $\theta=(1,1, \ldots, 1)^{T}$.

If the reduced system is observable, then the gradient $k^{i, j}$ of the linear asymptote, in terms of the original system matrices, is given by

$$
\begin{aligned}
& k^{i, j}=\min _{\zeta \in \mathbb{R}} \frac{1}{2}\left\|\mathcal{N}_{P}\left(\begin{array}{c}
B_{f}^{i} \\
D_{f}^{i}
\end{array}\right)-\zeta \mathcal{N}_{P}\left(\begin{array}{c}
B_{f}^{j} \\
D_{f}^{j}
\end{array}\right)\right\|^{2}= \\
& \min _{\mathbf{x}, \zeta} \frac{1}{2}\left(\left\|\mathbf{x}-A \mathbf{x}+\zeta B_{f}^{j}-B_{f}^{i}\right\|^{2}+\left\|C \mathbf{x}+D_{f}^{i}-\zeta D_{f}^{j}\right\|^{2}\right)
\end{aligned}
$$

The procedure to compute $k^{i, j}$ in the case when the reduced system is not observable is to first derive the reduced system with respect to $j$, as described in Section 4 , then remove the non-observable part, and finally compute $k^{i}$ according to Theorem 1 for the observable subsystem.

The following asymptotic result explains the nonsymmetry observed in Section 2, i.e., that it easier to isolate $f^{2}$ from $f^{3}$ than vice versa, and that this is a consequence of the fact that it is easier to detect $f^{2}$ than $f^{3}$ :

Corollary 1 If the reduced models, with respect to $f^{i}$ and $f^{j}$, are both observable, then $\frac{k^{i, j}}{k^{j, i}}=\frac{k^{i}}{k^{j}}$, and it follows that

$$
\lim _{N \rightarrow \infty} \frac{\mathcal{D}^{i, j}(\theta ; N)}{\mathcal{D}^{j, i}(\theta ; N)}=\lim _{N \rightarrow \infty} \frac{\mathcal{D}^{i}(\theta ; N)}{\mathcal{D}^{j}(\theta ; N)} .
$$

This corollary follows from the geometric interpretation in Figure 2 showing the relation between $k^{i}$ given by (8), and $k^{i, j}$, given by (10). The relation is $k^{i, j}=k^{i} \sin ^{2} \alpha$ and it can be seen that distinguishability increases when $\sin \alpha$ increases.

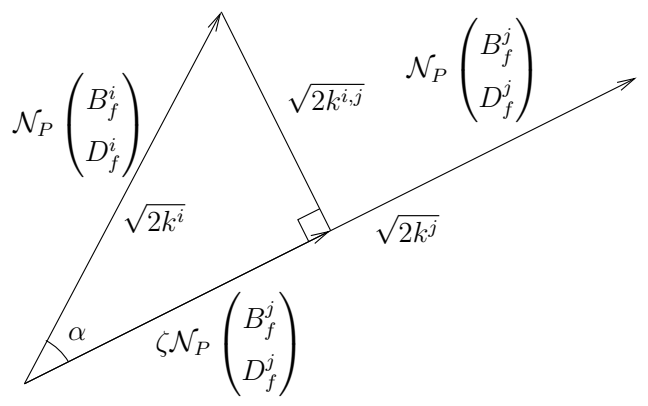

Fig. 2. Geometric interpretation of the relation between the gradients $k^{i}, k^{j}$, and $k^{i, j}$, with the $\zeta$ that minimizes (10). 


\section{The Least Squares Problem}

In the forthcoming sections, the asymptotic behavior of least squares problem in the form (5) will be studied, and now a procedure to transform the least squares problem (4) into the form (5) will be presented. The procedure is divided into three cases, which together cover all possible combinations.

Case 1: $B_{f}^{j}=0$ and $D_{f}^{j} \neq 0$. After some row operations on the measurement equations, it can be assumed that the fault is affecting a single sensor, and computing $\mathcal{D}^{i, j}$, is reduced to computing $\mathcal{D}^{i}$ for the model where the measurement equation with the faulty sensor has been removed. For example, if we want to compute $\mathcal{D}^{2,1}$ for the model (3), i.e., isolate fault $f^{2}$ from fault $f^{1}$, then the model is reduced by removing the first measurement equation and the fault $f^{3}$ from the model.

Case 2: $B_{f}^{j} \neq 0$ and $D_{f}^{j} \neq 0$. This second case can be reduced to the first case, by using the measurement equations to eliminate the fault $f^{j}$ from the dynamic equations. Note that this means that the measurement signal will be included in the dynamic equation, but this is not a problem since it is a known signal and it is not necessary to distinguish between known measurement and control signals in the analysis. For example, to compute $\mathcal{D}^{1,2}$ for the model (3), i.e. isolate from $f^{2}$, the fault $f^{2}$ is eliminated from the first dynamic equation by using the second measurement equation, which is then removed.

Case 3: $B_{f}^{j} \neq 0$ and $D_{f}^{j}=0$. In the third and remaining case the fault is included only in the dynamic equations. It can be assumed that the fault is included in only one equation, after a change of variables, and minimizing with respect to $\theta^{j}$, in (4), is equivalent to removing that equation. However, since a dynamic equation was removed, the system is no longer in state space form, which will be assumed in the asymptotic analysis later on. If the dynamic equation for $x_{t}^{k}$ is removed, then the reduced least square problem has the same structure as the original problem (4), with the role of $\theta_{t}^{j}$ replaced by $x_{t}^{k}$, which now can be treated in the same way as a fault in the least squares problem.

For example, consider $\mathcal{D}^{2,3}$ for the model (3). Then the second dynamic equation contains the fault $f^{3}$, and this equation is therefore removed. The role of the fault $f_{t}^{3}$ is replaced by the state $x_{t}^{2}$, which is included in the second measurement equation. The problem has been reduced to the first case and second measurement equation is therefore removed as before. The problem has been reduced to computing $\tilde{\mathcal{D}}^{2}(\theta, N)$ for the reduced model.

In Theorem 1 and Theorem 2 it is assumed that the fault time profile is $\theta=(1,1, \ldots, 1)^{T}$, and this assumption is assumed to be fulfilled in the remaining part of the paper. In this case the least squares problem (5) can be written as

$\mathcal{D}^{i}(\theta ; N)=\min _{\mathbf{x}_{t}} \frac{1}{2} \sum_{t=1}^{N}\left\|\mathbf{x}_{t+1}-A \mathbf{x}_{t}-\mathbf{b}\right\|^{2}+\left\|C \mathbf{x}_{t}+\mathbf{d}\right\|^{2}$,

where $\mathbf{b}=B_{f}^{i}$ and $\mathbf{d}=D_{f}^{i}$. The normal equations are derived by setting the gradients, with respect to $\mathbf{x}_{t}$, of the objective function in (12) equal to zero. By defining $X_{t}=$ $\left(\mathbf{x}_{t+1}^{T} \mathbf{x}_{t}^{T}\right)^{T}$ the normal equations can be formulated as a discrete two point boundary value problem:

$$
\begin{aligned}
X_{t} & =T X_{t-1}+B, \\
G_{1} X_{1}+H_{1} & =0, \\
G_{N} X_{N}+H_{N} & =0,
\end{aligned}
$$

where

$$
\begin{aligned}
T & =\left(\begin{array}{cc}
T_{1} & T_{2} \\
I & 0
\end{array}\right), \quad B=\left(\begin{array}{c}
A^{-T}\left(\left(A^{T}-I\right) \mathbf{b}+C^{T} \mathbf{d}\right) \\
0
\end{array}\right), \\
T_{1} & =A+A^{-T}+A^{-T} C^{T} C, \quad T_{2}=-A^{-T} A, \\
G_{1} & =\left(I-A-A^{-T} C^{T} C\right), \quad H_{1}=-\mathbf{b}-A^{-T} C^{T} \mathbf{d}, \\
G_{N} & =(I-A), \quad H_{N}=-\mathbf{b} .
\end{aligned}
$$

The first equation (13a) corresponds to the gradients w.r.t. $\mathbf{x}_{t}$ for $t=2, \ldots, N$, and (13b), (13c) correspond to the gradients w.r.t. $\mathbf{x}_{1}$ and $\mathbf{x}_{N+1}$, respectively

\section{$5 \quad$ Asymptotics of the minimizing sequence}

In this section, the two point boundary value problem (13a), (13b), and (13c), will be studied and asymptotic results will be derived for the solution $\mathbf{x}_{k}$, i.e., the minimizing sequence of the least squares problem (12). This is the first step in the derivation of the asymptotics of the distinguishability measure, i.e., the minimal value of the objective function in (12). The main result of this section is the following.

Theorem 3 If the system (1) is observable, then there exists a unique solution $\mathbf{x}_{t}, t=1, \ldots, N+1$ of the minimization problem (12). Furthermore, there exist constants $0<\varrho<1$ and $c>0$, such that for any $N$, the solution $\mathbf{x}_{t}, t=1, \ldots, N+1$, fulfills the inequality

$$
\left\|\mathbf{x}_{t}-\left(\mathbf{x}^{\dagger}+\mathbf{x}_{1, t}+\mathbf{x}_{\infty, N+2-t}\right)\right\| \leq c \varrho^{N},
$$

where $\mathbf{x}^{\dagger}$ is defined by

$$
\mathbf{x}^{\dagger}=\underset{\mathbf{x}}{\arg \min } \frac{1}{2}\left(\|\mathbf{x}-A \mathbf{x}-\mathbf{b}\|^{2}+\|C \mathbf{x}+\mathbf{d}\|^{2}\right),
$$


and $\mathbf{x}_{1, t}, \mathbf{x}_{\infty, t}, t=1,2, \ldots$, are infinite sequences of vectors that satisfy the inequalities

$$
\left\|\mathbf{x}_{1, t}\right\| \leq c \varrho^{t}, \quad\left\|\mathbf{x}_{\infty, t}\right\| \leq c \varrho^{t}
$$

for $t=1,2, \ldots$

Remark 1 In this theorem and throughout the paper, $c$ will be used to denote arbitrary small positive constants that only depend on system properties.

This theorem will be proved by using asymptotic methods developed for boundary value problems applied to the normal equations (13a), (13b), and (13c); see, e.g., [5]. The approach can be divided into two main steps. First a sequence $S_{t}^{(1)}$ is constructed in such a way that it fulfills the two point boundary value problem (13a), (13b), and (13c) approximately in the sense that the approximation satisfies the boundary value problem with an error that tends to zero as $N$ tends to infinity. Then it is shown that this implies that the sequence $S_{t}^{(1)}$ is an asymptotic approximation of the solution $X_{t}$, by estimating the remainder $X_{t}-S_{t}^{(1)}$.

\subsection{The approximate solution of the boundary value problem}

As a first approximate solution of the boundary value problem, the sequence $X_{t}^{(0)}=\left(\mathbf{x}^{\dagger T} \mathbf{x}^{\dagger T}\right)^{T}, t=1, \ldots, N$, is chosen, where $\mathbf{x}^{\dagger}$ is defined by (15). It is straightforward to check that the normal equations

$$
\left(\begin{array}{c}
A-I \\
C
\end{array}\right)^{T}\left(\left(\begin{array}{c}
A-I \\
C
\end{array}\right) \mathbf{x}^{\dagger}+\left(\begin{array}{l}
\mathbf{b} \\
\mathbf{d}
\end{array}\right)\right)=0
$$

for the least squares problem (15) are equivalent to $X_{t}^{(0)}=T X_{t-1}^{(0)}+B$. Hence, $X_{t}^{(0)}$ fulfills equation (13a).

Now, the boundary conditions are considered. By inserting the approximation $X^{(0)}$ in the first condition (13b), the expression $E_{1}^{(0)}=G_{1} X_{1}^{(0)}+H_{1}$ for the error is obtained. To compensate for this error, the boundary layer sequence $X_{t}^{(1)}=\left(\mathbf{x}_{t+1}^{(1) T} \mathbf{x}_{t}^{(1) T}\right)^{T}$ defined by the recursive relation

$$
\begin{aligned}
X_{t}^{(1)} & =T X_{t-1}^{(1)}, t=2, \ldots, N, \\
G_{1} X_{1}^{(1)} & =-E_{1}^{(0)}, \\
Q_{1} X_{1}^{(1)} & =0
\end{aligned}
$$

is added to the approximation $X^{(0)}$. The first equation, (17a), is the homogeneous recursive relation. The boundary condition $(17 \mathrm{~b})$ is chosen so that the boundary layer will compensate for the error $E_{1}^{(0)}$. The purpose of the second boundary condition (17c), where $Q_{1}$ is an $n \times 2 n$ matrix to be defined in the next section, is to assure that the sequence $X_{t}^{(1)}=T^{t-1} X_{1}^{(1)}$ tends to zero as $t$ tends to infinity in order to make the error caused in the other boundary condition (13c) as small as possible. In Lemma 3, it will be shown that there exists a unique solution $X_{1}^{(1)}$ of the initial condition above and the sequence is hence well-defined.

By inserting the approximation $X_{t}^{(0)}$ in the second condition (13c), the error $E_{N}^{(0)}=G_{N} X_{N}^{(0)}+H_{N}$ is obtained and the corresponding boundary layer $X_{t}^{(-1)}=$ $\left(\mathbf{x}_{t+1}^{(-1) T} \mathbf{x}_{t}^{(-1) T}\right)^{T}$ is defined similarly by

$$
\begin{aligned}
X_{t-1}^{(-1)} & =T^{-1} X_{t}^{(-1)}, t=N, \ldots, 2 \\
G_{N} X_{N}^{(-1)} & =-E_{N}^{(0)}, \quad Q_{N} X_{N}^{(-1)}=0 .
\end{aligned}
$$

Now, the two boundary layer sequences are added to the approximation to obtain

$$
S_{t}^{(1)}=\left(\begin{array}{c}
\mathbf{s}_{t+1}^{(1)} \\
\mathbf{s}_{t}^{(1)}
\end{array}\right)=X_{t}^{(0)}+X_{t}^{(1)}+X_{t}^{(-1)} .
$$

\subsection{Asymptotic behaviour of the boundary layers}

The boundary layers above were defined recursively using the operators $T$ and $T^{-1}$ and the objective of this section is to derive conditions on a vector $X$ such that $T^{t} X$ or $T^{-t} X$ tends to zero as $t$ tends to infinity. A first step is to distinguish between eigenvalues with modulus less and greater than one, respectively.

Lemma 1 If the system is observable, then there are $n$ eigenvalues $\lambda$ of the matrix $T$ such that $0<|\lambda|<1$ and $n$ eigenvalues $\lambda$ such that $1<|\lambda|$. The eigenvalues $\lambda_{i}$, $i=1, \ldots, 2 n$, can be enumerated so that $\left|\lambda_{i}\right|>1$ and $\lambda_{i+n}=\lambda_{i}^{-1}$ for $i=1, \ldots, n$.

PROOF. First, it follows from the definition of $T$ and the assumption that $A$ is invertible that $\operatorname{det} T=\operatorname{det}\left(A^{-T} A\right)=1 \neq 0$. Hence $\lambda=0$ is not an eigenvalue of $T$. The characteristic polynomial of $T$ is given by

$$
\begin{aligned}
p(\lambda) & =\operatorname{det}(T-\lambda I)=\operatorname{det}\left(\begin{array}{cc}
T_{1}-\lambda I & T_{2} \\
I & -\lambda I
\end{array}\right) \\
& =\operatorname{det}\left(\begin{array}{cc}
T_{1}-\lambda I & I \\
I & 0
\end{array}\right)\left(\begin{array}{cc}
I & -\lambda I \\
0 & -\lambda^{2} I+\lambda T_{1}+T_{2}
\end{array}\right) \\
& =\operatorname{det}\left(\lambda^{2} I-\lambda T_{1}-T_{2}\right) \\
& =\operatorname{det}\left(\lambda^{2} I-\lambda\left(A+A^{-T}+A^{-T} C^{T} C\right)+A^{-T} A\right) .
\end{aligned}
$$


Now, it will be shown that there is no eigenvalue $\lambda$ such that $|\lambda|=1$. Assume that $|\lambda|=1$. Then $\lambda^{-1}=\bar{\lambda}$ and

$$
\begin{aligned}
& p(\lambda)=\operatorname{det}\left(\lambda^{2} I-\lambda\left(A+A^{-T}+A^{-T} C^{T} C\right)+A^{-T} A\right) \\
& =\operatorname{det}\left(-\lambda A^{-T}\right) \operatorname{det}\left(-\lambda A^{T}+\left(A^{T} A+I+C^{T} C\right)-\bar{\lambda} A\right) \\
& =\operatorname{det}\left(-\lambda A^{-T}\right) \operatorname{det}\left(\left(\begin{array}{c}
A-\lambda I \\
C
\end{array}\right)^{*}\left(\begin{array}{c}
A-\lambda I \\
C
\end{array}\right)\right) \neq 0 .
\end{aligned}
$$

The last inequality follows from the fact that the matrix $\left((A-\lambda I)^{T} C^{T}\right)^{T}$ has full column rank by the PBH condition for observability; see Section 5.3 in [6].

Let's consider the characteristic polynomial again. By factoring out $\lambda^{2} A^{-T}$ from the matrix expression, $p(\lambda)$ can be rewritten as

$\lambda^{2 n} \operatorname{det}\left(A^{-T}\right) \operatorname{det}\left(A^{T}-\lambda^{-1}\left(A^{T} A+I+C^{T} C\right)+\lambda^{-2} A\right)$.

By transposing the matrix in the second determinant and then multiply with matrix in the first determinant, the relation $p(\lambda)=\lambda^{2 n} p(1 / \lambda)$ is obtained. Hence if $\lambda$ is an eigenvalue of $T$, then $1 / \lambda$ is an eigenvalue as well with the same algebraic multiplicity. This concludes the proof.

From now on it will be assumed that the eigenvalues are enumerated as in Lemma 1 and a constant $\varrho$ is chosen so that

$$
\max _{n+1 \leq i \leq 2 n}\left|\lambda_{i}\right|<\varrho<1 .
$$

It follows that $\varrho<\left|\lambda_{i}\right|$, for $i=1, \ldots, n$, and $\left|\lambda_{i}\right|^{-1}<\varrho$, for $i=n+1, \ldots, 2 n$. Using the Jordan normal form, see e.g. Chapter 1 in [6], the matrix $T$ can be written as $T=P \tilde{T} P^{-1}$, where $\tilde{T}=\operatorname{diag}\left(\tilde{T}_{1}, \tilde{T}_{N}\right)$ and where $\tilde{T}_{1}$ contains the Jordan blocks corresponding to the eigenvalues $\lambda_{1}, \ldots, \lambda_{n}$ and $\tilde{T}_{N}$ contains the Jordan blocks corresponding to $\lambda_{n+1}, \ldots, \lambda_{2 n}$. It follows from Lemma 1 that $\tilde{T}_{1}$ and $\tilde{T}_{N}$ are both square matrices of order $n$. Partition $P^{-1}=\left(Q_{1}^{T} Q_{N}^{T}\right)^{T}$, where $Q_{1}$ and $Q_{N}$ have $n$ rows. The main result of this subsection is the following.

Lemma 2 If the system is observable, then the inequalities

$$
\begin{gathered}
\max _{X \neq 0, Q_{N} X=0} \frac{\left\|T^{-t} X\right\|}{\|X\|} \leq c \varrho^{t}, \\
\max _{X \neq 0, Q_{1} X=0} \frac{\left\|T^{t} X\right\|}{\|X\|} \leq c \varrho^{t},
\end{gathered}
$$

hold for $t=1,2, \ldots$

PROOF. If $X$ fulfills the condition $Q_{N} X=0$, then

$$
T^{-t} X=P\left(\begin{array}{c}
\tilde{T}_{1}^{-t} X \\
0
\end{array}\right)
$$

The matrix $\tilde{T}_{1}^{-t}$ is a block diagonal matrix with powers of the inverses of the Jordan blocks, corresponding to the eigenvalues fulfilling $\left|\lambda_{i}\right|>1$, in the main diagonal. It is straightforward to show that the elements of the these blocks are bounded from above by $c\left|\lambda_{i}\right|^{-t} t^{l}$, where $l$ is the length of the longest Jordan chain, and it follows that $\left\|T^{-t} X\right\| \leq c \varrho^{t}\|X\|$ which proves inequality (18). Inequality (19) can be obtained in the same way.

Now, the remainder sequence

$$
R_{t}=\left(\begin{array}{c}
\mathbf{r}_{t+1} \\
\mathbf{r}_{t}
\end{array}\right)=X_{t}-S_{t}^{(1)}, \quad t=1, \ldots, N
$$

will be analyzed. From the definitions of $X_{t}$ and $S_{t}^{(1)}$ it follows that the sequence $R_{t}$ fulfills the equations

$$
\begin{aligned}
R_{t} & =T R_{t-1}, t=2, \ldots, N \\
G_{1} R_{1} & =-E_{1}^{(1)}, \quad G_{N} R_{N}=-E_{N}^{(1)} .
\end{aligned}
$$

where $E_{1}^{(1)}=G_{1} T^{1-N} X_{N}^{(-1)}$ and $E_{N}^{(1)}=G_{N} T^{N-1} X_{1}^{(1)}$ are the errors in the boundary conditions. Note that $X_{N}^{(-1)}$ and $X_{1}^{(1)}$ do not depend on $N$ and fulfill $Q_{1} X_{1}^{(1)}=$ $Q_{N} X_{N}^{(-1)}=0$. Hence, it follows from Lemma 2 that

$$
\left\|E_{1}^{(1)}\right\| \leq c \varrho^{N}, \quad\left\|E_{N}^{(1)}\right\| \leq c \varrho^{N} .
$$

\subsection{Estimate of the remainder}

The boundary layer sequences in Theorem 3 can now be defined as

$$
\mathbf{x}_{1, t}=\mathbf{x}_{t}^{(1)}, \quad \mathbf{x}_{\infty, t}=\mathbf{x}_{N+2-t}^{(-1)}, \quad t=1, \ldots, N+1 .
$$

It follows from the definitions of $X_{t}^{(1)}$ and $X_{t}^{(-1)}$ that the elements in the sequences do not depend on $N$ and can thus be extended to infinite sequences, and with this notation, it follows that

$$
\mathbf{r}_{t}=\mathbf{x}_{t}-\left(\mathbf{x}^{\dagger}+\mathbf{x}_{1, t}+\mathbf{x}_{\infty, N+2-t}\right)
$$

Now the proof of Theorem 3 is given.

\section{PROOF.}

There exists a unique solution of the least squares problem (5) if and only if the matrix $H$ in (2) has full rank and it can be shown that the system is observable if and only if the matrix $H$ has full column rank for $N \geq n$, see Section 2.6 in [6]. This proves the first statement. 
The inequalities (16) follow from Lemma 2. Now the inequality

$$
\left(\sum_{t=1}^{N+1}\left\|\mathbf{r}_{t}\right\|^{2}\right)^{1 / 2} \leq c \varrho^{N}
$$

will be shown, which implies estimate (14) in Theorem 3. Using the definitions of $T, G_{1}$, and $G_{N}$, the equations (21) can be rewritten as

$$
\begin{aligned}
\mathbf{r}_{t}-A \mathbf{r}_{t-1}-A^{T}\left(\mathbf{r}_{t+1}-A \mathbf{r}_{t}\right)+C^{T} C \mathbf{r}_{t} & =0, t=2, \ldots, N, \\
-A^{T}\left(\mathbf{r}_{2}-A \mathbf{r}_{1}\right)+C^{T} C \mathbf{r}_{1} & =-E_{1}^{(1)}, \\
\mathbf{r}_{N+1}-A \mathbf{r}_{N} & =-E_{N}^{(1)}
\end{aligned}
$$

Multiplying these equations above with $\mathbf{r}_{t}^{T}, \mathbf{r}_{1}^{T}$, and $\mathbf{r}_{N+1}^{T}$, respectively, from the left and summing with respect to $t$, the relation

$$
\sum_{t=1}^{N}\left\|\mathbf{r}_{t+1}-A \mathbf{r}_{t}\right\|^{2}+\left\|C \mathbf{r}_{t}\right\|^{2}=-\mathbf{r}_{1}^{T} E_{1}^{(1)}-\mathbf{r}_{N+1}^{T} E_{N}^{(1)}
$$

is obtained. The matrix $H$ has full rank if $N=n$, see Section 2.6 in [6]. This implies $\left\|\mathbf{r}_{b}\right\| \leq c\left\|H \mathbf{r}_{b}\right\|$ for any vector $\mathbf{r}_{b}$, and

$$
\sum_{t=1}^{N+1}\left\|\mathbf{r}_{t}\right\|^{2} \leq c \sum_{t=1}^{N}\left\|\mathbf{r}_{t+1}-A \mathbf{r}_{t}\right\|^{2}+\left\|C \mathbf{r}_{t}\right\|^{2}
$$

for $N=n$. By using this estimate repeatedly, it can be shown that the same inequality holds for any $N \geq$ $n$, where the constant does not depend on $N$. CauchySchwarz inequality, together with (22), gives

$$
\begin{aligned}
-\mathbf{r}_{1}^{T} E_{1}^{(1)}-\mathbf{r}_{N+1}^{T} E_{N}^{(1)} \leq c & \left(\left\|\mathbf{r}_{1}\right\|+\left\|\mathbf{r}_{N+1}\right\|\right) \varrho^{N} \\
& \leq c \varrho^{N}\left(\sum_{t=0}^{N+1}\left\|\mathbf{r}_{t}\right\|^{2}\right)^{1 / 2}
\end{aligned}
$$

This inequality, together with (24) and (25), implies estimate (23) for the remainder. This concludes the proof of Theorem 3 .

What remains now is to show that the boundary conditions in the definitions of $X_{t}^{(1)}$ and $X_{t}^{(-1)}$ can always be fulfilled.

Lemma 3 The matrices $\left(G_{1}^{T} Q_{1}^{T}\right)^{T}$, and $\left(G_{N}^{T} Q_{N}^{T}\right)^{T}$, are non-singular.

PROOF. Assume that the vector $R_{1}^{\prime}$ is in the null space of the first matrix, i.e. $G_{1} R_{1}^{\prime}=Q_{1} R_{1}^{\prime}=0$. Define $R_{t}^{\prime}=T R_{t-1}^{\prime}, t=2, \ldots, N$. It follows from the condition $Q_{1} R_{1}^{\prime}=0$ that the sequence satisfies the inequality $\left\|G_{N} R_{N}^{\prime}\right\| \leq c \varrho^{N}$ for all $N$.
It follows from the relations above that $R_{t}^{\prime}$ satisfies the equations (21), where $E_{1}^{(1)}=0$ and $E_{N}^{(1)}$ satisfies the second estimate in (22), and it follows, in the same way as in the proof Theorem 3, that $R_{t}^{\prime}$ satisfies the same estimate as $R_{t}$. In particular $\left\|R_{1}^{\prime}\right\| \leq c \varrho^{N}$ for all $N$ and it follows that $R_{1}^{\prime}=0$, i.e., the homogeneous equation only has the trivial solution, which proves that the first matrix in is non-singular. The corresponding result for the second matrix can be shown in the same way.

\section{Proofs of the Theorems in Section 3}

The basic idea in the first proof is to substitute the asymptotic approximation of the solution of the least squares problem, given by Theorem 3, into the corresponding objective function to obtain the asymptotic results for the distinguishability measure. The details are given in the following proof of Theorem 1:

PROOF. Using the inequality

$$
\left|\|\mathbf{x}\|^{2}-\|\mathbf{y}\|^{2}\right| \leq(\|\mathbf{x}\|+\|\mathbf{y}\|)\|\mathbf{x}-\mathbf{y}\|
$$

together with inequality (23), $\mathbf{r}_{t}=\mathbf{x}_{t}-\mathbf{s}_{t}$ and

$$
k=\frac{1}{2}\left(\left\|\mathbf{x}^{\dagger}-A \mathbf{x}^{\dagger}-\mathbf{b}\right\|^{2}+\left\|C \mathbf{x}^{\dagger}+\mathbf{d}\right\|^{2}\right)
$$

the relation

$$
\begin{aligned}
\frac{1}{2} \sum_{t=1}^{N} & \left\|\mathbf{x}_{t+1}-A \mathbf{x}_{t}-\mathbf{b}\right\|^{2}+\left\|C \mathbf{x}_{t}+\mathbf{d}\right\|^{2} \\
= & \frac{1}{2} \sum_{t=1}^{N}\left(\left\|\mathbf{s}_{t+1}^{(1)}-A \mathbf{s}_{t}^{(1)}-\mathbf{b}\right\|^{2}-\left\|\mathbf{x}^{\dagger}-A \mathbf{x}^{\dagger}-\mathbf{b}\right\|^{2}\right. \\
& \left.+\left\|C \mathbf{s}_{t}^{(1)}+\mathbf{d}\right\|^{2}-\left\|C \mathbf{x}^{\dagger}+\mathbf{d}\right\|^{2}\right) \\
& +k N+\mathcal{O}\left(\varrho^{N}\right) .
\end{aligned}
$$

can be obtained. Using first $s_{t}=\mathbf{x}^{\dagger}+\mathbf{x}_{1, t}+\mathbf{x}_{\infty, N+2-t}$ together with the second inequality in (16), and then inequality (26), together with the first inequality in (16), the following relation can be obtained

$$
\begin{aligned}
& \frac{1}{2} \sum_{t=1}^{\lfloor N / 2\rfloor}\left\|C \mathbf{s}_{t}^{(1)}+\mathbf{d}\right\|^{2}-\left\|C \mathbf{x}^{\dagger}+\mathbf{d}\right\|^{2} \\
= & \frac{1}{2} \sum_{t=1}^{\lfloor N / 2\rfloor}\left(\left\|C\left(\mathbf{x}^{\dagger}+\mathbf{x}_{1, t}\right)+\mathbf{d}\right\|^{2}-\left\|C \mathbf{x}^{\dagger}+\mathbf{d}\right\|^{2}\right)+\mathcal{O}\left(\varrho^{N / 2}\right) \\
= & \frac{1}{2} \sum_{t=1}^{\infty}\left(\left\|C\left(\mathbf{x}^{\dagger}+\mathbf{x}_{1, t}\right)+\mathbf{d}\right\|^{2}-\left\|C \mathbf{x}^{\dagger}+\mathbf{d}\right\|^{2}\right)+\mathcal{O}\left(\varrho^{N / 2}\right) .
\end{aligned}
$$


where $\lfloor N / 2\rfloor$ denotes the integer part of $N / 2$. Using the same approach on the other parts of the sum (27) the following result is obtained.

$\frac{1}{2} \sum_{t=1}^{N}\left\|\mathbf{x}_{t+1}-A \mathbf{x}_{t}-\mathbf{b}\right\|^{2}+\left\|C \mathbf{x}_{t}+\mathbf{d}\right\|^{2}=k N+m+\mathcal{O}\left(\varrho^{N / 2}\right)$

where the constant term is given by

$$
\begin{aligned}
m= & \frac{1}{2} \sum_{t=1}^{\infty}\left\|\mathbf{x}^{\dagger}+\mathbf{x}_{1, t+1}-A\left(\mathbf{x}^{\dagger}+\mathbf{x}_{1, t}\right)-\mathbf{b}\right\|^{2} \\
& -\left\|\mathbf{x}^{\dagger}-A \mathbf{x}^{\dagger}-\mathbf{b}\right\|^{2} \\
& +\left\|\mathbf{x}^{\dagger}+\mathbf{x}_{\infty, t}-A\left(\mathbf{x}^{\dagger}+\mathbf{x}_{\infty, t+1}\right)-\mathbf{b}\right\|^{2} \\
& -\left\|\mathbf{x}^{\dagger}-A \mathbf{x}^{\dagger}-\mathbf{b}\right\|^{2} \\
& +\left\|C\left(\mathbf{x}^{\dagger}+\mathbf{x}_{1, t}\right)+\mathbf{d}\right\|^{2}-\left\|C \mathbf{x}^{\dagger}+\mathbf{d}\right\|^{2} \\
& +\left\|C\left(\mathbf{x}^{\dagger}+\mathbf{x}_{\infty, t+1}\right)+\mathbf{d}\right\|^{2}-\left\|C \mathbf{x}^{\dagger}+\mathbf{d}\right\|^{2}
\end{aligned}
$$

which proves (6). Finally (8) follows from Lemma 1 in [2].

It will now be shown how an explicit expression can be derived for the constant term $m$ in Theorem 1 in terms of eigenvalues and eigenvectors of the operator $T$. It is assumed, for the sake of simplicity, that $T$ is diagonalizable. The result can be extended to the nondiagonalizable case, but the expression will be more complicated and is therefore omitted.

It follows from the conditions $Q_{1} X_{1}^{(1)}=0$ and $Q_{N} X_{N}^{(-1)}=0$, that $X_{1}^{(1)} \in \operatorname{span}\left\{P_{n+1}, \ldots, P_{2 n}\right\}$ and $X_{N}^{(1)} \in \operatorname{span}\left\{P_{n 1}, \ldots, P_{n}\right\}$, where $P_{i}$ is the eigenvector of $T$ corresponding to the eigenvalue $\lambda_{i}$ for $i=n+1, \ldots, 2 n$. Hence,

$$
X_{1}^{(1)}=\sum_{i=n+1}^{2 n} \mu_{i} P_{i}, \quad X_{N}^{(-1)}=\sum_{i=1}^{n} \mu_{i} P_{i}
$$

for some coefficients $\mu_{i}, i=1, \ldots, 2 n$. It follows from the recursive definition of $X_{t}^{(1)}$ and $X_{t}^{(-1)}$ that

$$
\mathbf{x}_{1, t}=\sum_{i=n+1}^{2 n} \lambda_{i}^{t-1} \mu_{i} \mathbf{y}_{i}, \quad \mathbf{x}_{\infty, t}=\sum_{i=1}^{n} \lambda_{i}^{-t+1} \mu_{i} \mathbf{y}_{i}
$$

where $\mathbf{y}_{i}$ are the first $n$ elements of $P_{i}$ for $i=1, \ldots, n$ and last $n$ elements of $P_{i}$ for $i=n+1, \ldots, 2 n$. By substituting (30) into (28), with the squared norms replaced by scalar products, and summing the geometric series the relation

$$
\begin{aligned}
& m=m_{1}-m_{0} \text { is obtained where } \\
& m_{l}=\sum_{i, j=1+l n}^{n+n l} \frac{\mu_{i} \mu_{j}}{1-\lambda_{i} \lambda_{j}}\left(\left(\lambda_{i} \mathbf{y}_{i}-A \mathbf{y}_{i}\right)^{T}\left(\lambda_{i} \mathbf{y}_{i}-A \mathbf{y}_{i}\right)\right. \\
& \left.+\left(C \mathbf{y}_{i}\right)^{T} C \mathbf{y}_{j}\right) \\
& +\sum_{i=1+l n}^{n+l n} \frac{2 \mu_{i}}{1-\lambda_{i}}\left(\left(\mathbf{x}^{\dagger}-A \mathbf{x}^{\dagger}-\mathbf{b}\right)^{T}\left(\lambda_{i} \mathbf{y}_{i}-A \mathbf{y}_{i}\right)\right. \\
& \left.\quad+\left(C \mathbf{x}^{\dagger}+\mathbf{d}\right)^{T} C \mathbf{y}_{i}\right), \quad l=0,1 .
\end{aligned}
$$

with $\lambda_{i}, \mu_{i}$, and $\mathbf{y}_{i}$ as described above.

The section is completed with the proof of Theorem 2:

PROOF. The asymptotic representation (6) in Theorem 2 follows from Theorem 1 and the fact that distinguishability in this case can be reduced to a detection problem, as outlined at the end of Section 3.

To prove the second part, assume that the reduced problem is observable. In this case Theorem 3 can be applied directly and the asymptotic representation in the form

$$
\mathbf{x}_{t}=\mathbf{x}^{\dagger}+\mathbf{x}_{1, t}+\mathbf{x}_{\infty, N+2-t}+\mathcal{O}\left(\varrho^{N}\right)
$$

is obtained for the remaining states of the reduced problem, where the terms are described in Theorem 3.

However, it follows from the reduction procedure, described in Section 4, that the eliminated faults and states of the original system is a linear combination of the remaining states, and therefore admits an asymptotic representation in the same form as (31).

A consequence of this is that the minimizing sequence in (4) can be approximated by $\mathbf{x}_{t}=\tilde{\mathbf{x}}^{\dagger}$, for a constant vector $\tilde{\mathbf{x}}^{\dagger}$, outside neighbourhoods of the end points. It follows that the principal term of $\mathcal{D}^{i, j}(\theta, N)$ as $N$ tends to infinity, i.e. $k^{i, j} N$, is given by

$\min _{\mathbf{x}, \zeta} \frac{N}{2}\left(\left\|\mathbf{x}-A \mathbf{x}+\zeta B_{f}^{j}-B_{f}^{i}\right\|^{2}+\left\|C \mathbf{x}+D_{f}^{i}-\zeta D_{f}^{j}\right\|^{2}\right)$

which gives (11), and finally (10) is obtained by minimizing with respect to $\mathbf{x}$ in (11).

\section{Conclusions}

In many fault diagnosis applications, the negative impact of sensor noise and model uncertainties on fault detection and isolation performance cannot be neglected. The analysis of the asymptote of the distinguishability measure gives useful information about fault detection and isolation properties for a given model. The gradient 
of the asymptote is relevant since it gives information about how much fault detection performance will improve for each new sample of sensor data which is useful for, e.g., model analysis and sensor selection.

\section{References}

[1] Michèle Basseville, Igor V Nikiforov, et al. Detection of abrupt changes: theory and application, volume 104. Prentice Hall Englewood Cliffs, 1993.

[2] Daniel Eriksson, Erik Frisk, and Mattias Krysander. A method for quantitative fault diagnosability analysis of stochastic linear descriptor models. Automatica, 49(6):1591-1600, June 2013.

[3] Fouzi Harrou, Lionel Fillatre, and Igor Nikiforov. Anomaly detection/detectability for a linear model with a bounded nuisance parameter. Annual Reviews in Control, (0):-, 2014.

[4] Thomas Kailath. Linear systems, volume 1. Prentice-Hall Englewood Cliffs, NJ, 1980.

[5] Vladimir Maz'ya, Serguei Nazarov, and Boris Plamenevskij. Asymptotic theory of elliptic boundary value problems in singularly perturbed domains. Vol. I \& II, volume 111 \& 112 of Operator Theory: Advances and Applications. Birkhäuser Verlag, Basel, 2000.

[6] Howard Harry Rosenbrock. State-space and multivariable theory. 1970. 